\title{
A model of student's dilemma
}

\author{
Adam Lipowski ${ }^{1}$ and António L. Ferreira ${ }^{2}$ \\ ${ }^{1}$ Faculty of Physics, Adam Mickiewicz University, 61-614 Poznań, Poland \\ ${ }^{2}$ Department of Physics, University of Aveiro, 3810 Aveiro, Portugal
}

\begin{abstract}
Each year perhaps millions of young people face the following dilemma: should I continue my education or rather start working with already acquired skills. Right decision must take into account somebody's own abilities, accessibility to education institutions, competition, and potential benefits. A multi-agent, evolutionary model of this dilemma predicts a transition between stratified and homogeneous phases, evolution that diminishes fitness, fewer applicants per seat for decreased capacity of the university, and presence of poor students at élite universities.
\end{abstract}

Education system constitutes an important element of every modern society. The main factor that attract people to education institutions is the fact that a knowledgeable individual receives a certain 'pay-off' that might translate into a better salary, fame, or self-satisfaction. But to be educated and harvest potential benefits, an individual must invest certain amount of time and very often money. In addition, there is a risk related to this process. For example, one can fail at the exam or loose interest in an earlier chosen subject. Moreover, one has to consider that only a fraction of a society can get access to education institutions and competition is sometimes fierce. Thus, before deciding whether to be, e.g., a university applicant, one has to take into account the above factors as well as carefully evaluate his/her own abilities.

Since abilities are difficult to assess and the number of competitors is difficult to foresee, there is no a simple recipe on making the right decision. As a result predicting the functioning of an educational system is far from trivial. One problem, for example, is a possibility that a strong pressure will push all individuals toward being university applicants. Such a situation might be quite frustrating for the society as a whole since a large fraction of them is bound to fail. Perhaps it would be better if part of a society would give up educational benefits and abstain from applying. But can we expect that optimal decisions of individuals will imply a well-being of a society? Another problem, is to predict the number of university applicants. This number is of much concern not only to university planners but also to applicants themselves. The later ones are more concerned with the ratio of the number of applicants and the amount of applicants the university will accept, since it better reflects the actual competition. How are these numbers changing when the university e.g., reduces the amount of accepted candidates? Will this ratio, and at the same time competition, increase? Or, will this imply a better level of university students? Naively, one can expect that élite universities that accept only few best applicants should be able to keep a very high level. However, a large risk of failure might put off potential high-level applicants opening the door to lower-level applicants. It seems that modelling of educational systems so far is restricted to certain spe- cific topics [1]. Certainly, some insight into more basic properties of such complex problems would be desirable that hopefully could improve our educational systems.

In the present paper we introduce a multi-agent evolutionary model that describes some aspects of educational systems. Such an approach proved to be very successful in studying some other conflicting social situations, known as the so-called prisoner dilemma [2] providing thus an elegant game-theory illustration of reciprocal altruism [3]. In our model there are $N$ individuals of levels of abilities $l_{i}$, and 'confidence level' $p_{i}, i=1, \ldots, N$ $\left(0<l_{i}, p_{i}<1\right)$. An elementary step (a unit of time) of the dynamics of our model is made of four parts: decision making, payoff, removal, and reproduction. First, each individual decides, according to a stochastic rule specified below, whether to apply or not to the university. The university accepts applicants provided that their total number is not greater than $M$. If so, only $M$ best (i.e., those with the highest values of $l_{i}$ ) applicants are accepted. A successful applicant receives a payoff $s$, while the rejected ones the failure payoff $f$. Those who decided not to apply receive the give-up payoff $g$. In our context it is natural to examine only the case $f<g<s$. The received payoff determines fitness of an individual and thus its survival and reproduction chances. More specifically, $K$ individuals are removed from the population according to the rule that a randomly chosen individual $i$ that received a payoff $x_{i}(=f, g$ or $s)$ is removed with a probability $p\left(x_{i}\right)=\exp \left(-x_{i} / z\right)$ where $z=\frac{1}{N} \sum_{i=1}^{N} x_{i}$ is the average fitness of the population. Finally, we create $K$ new individuals reproducing surviving individuals. In particular, a selected survived individual produces an offspring with probability $1-\exp \left(-x_{i} / z\right)$. An offspring inherits parameters $l_{i}$ and $p_{i}$ of his parent subject to a small mutation of amplitude $\delta[4]$.

A decision of $i$-th individual whether to apply to the university or not is based on the comparison of his/her level $l_{i}$ with the average level $\bar{l}$ of successful applicants of the previous enrollment. The simplest rule might be for example to apply to the university only when $l_{i}>\bar{l}$ and not apply otherwise. However, we use another rule that allows for both choices to be made albeit with some probability that is specified by the 'confidence level' $p_{i}$. 


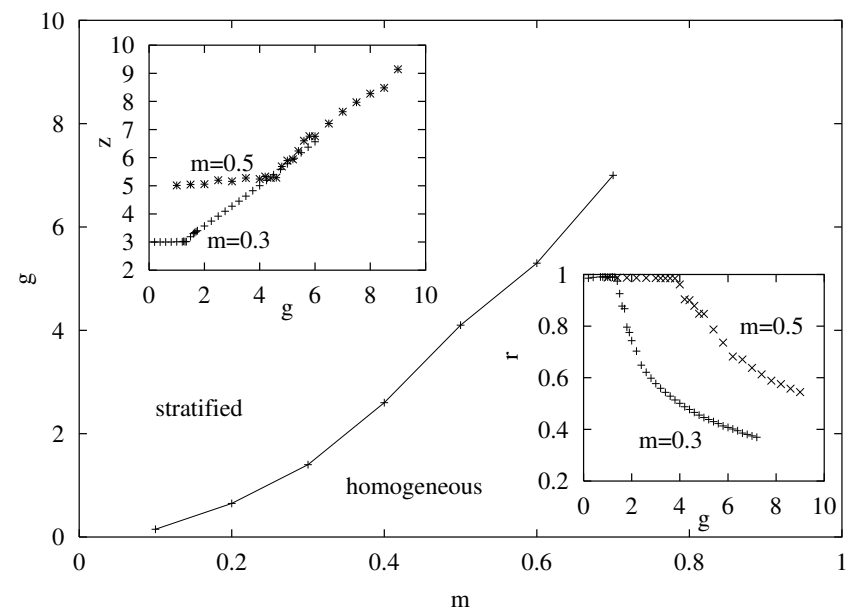

FIG. 1: Transition line in the $(g, m)$ plane separating homogeneous and stratified phases. Upper and lower insets show the fitness $z$, and average number of individuals that decided to apply to the university $r$, as functions of $g$.

In particular, an individual applies to the university with a probability $h\left(l_{i}, p_{i}\right)$ being a continuous piece-wise linear increasing function of $l_{i}$ and $p_{i}$ defined as

$$
h\left(l_{i}, p_{i}\right)= \begin{cases}p_{i} l_{i} / \bar{l} & \text { for } l_{i}<\bar{l} \\ {\left[\left(1-p_{i}\right) l_{i}-\bar{l}+p_{i}\right] /(1-\bar{l})} & \text { for } l_{i} \geq \bar{l}\end{cases}
$$

The function $h\left(l_{i}, p_{i}\right)$ has the property that $h\left(l_{i}=\right.$ $\left.0, p_{i}\right)=0, h\left(l_{i}=1, p_{i}\right)=1$ and $h\left(l_{i}=\bar{l}, p_{i}\right)=p_{i}$.

To examine the behaviour of our model we made numerical simulations. Presented results are obtained for $N=1000$ but we also made simulations for $N=$ 500,2000 and 5000 with qualitatively the same behaviour. We used payoff values $s=10, f=0$, the number of removed individuals $K=N / 10$, and the mutation amplitude $\delta=0.01$ (qualitatively the same behaviour was seen for $\delta=0.001$ ). Varying the give-up payoff $g$ and the normalized capacity of the university $m=M / N$, we monitored certain characteristics of our model such as the ratio $r$ equal to the number of individuals that decide to apply to the university divided by $N$, the average fitness $z$, and some probability distributions. Unless specified otherwise, simulations start with all individuals having $l_{i}$ and $p_{i}$ randomly chosen from the unit interval $(0,1)$. Stationary averages are calculated only after relaxing the system for sufficiently long time. Obtained results are described below [5].

First, numerical simulations show that the population with the initial uniform distribution of $l_{i}$ and $p_{i}$ quickly evolves toward much different states. Stationary behaviour, (see Fig. 1) depends on parameters $g$ and $m$. For sufficiently small $g$ or sufficiently large $m$ the system remains in the homogeneous phase where all individuals are clustered around $(1,1)$ point in the $\left(l_{i}, p_{i}\right)$ plane. In other words, the population consists of individuals of a very good level and which most likely will

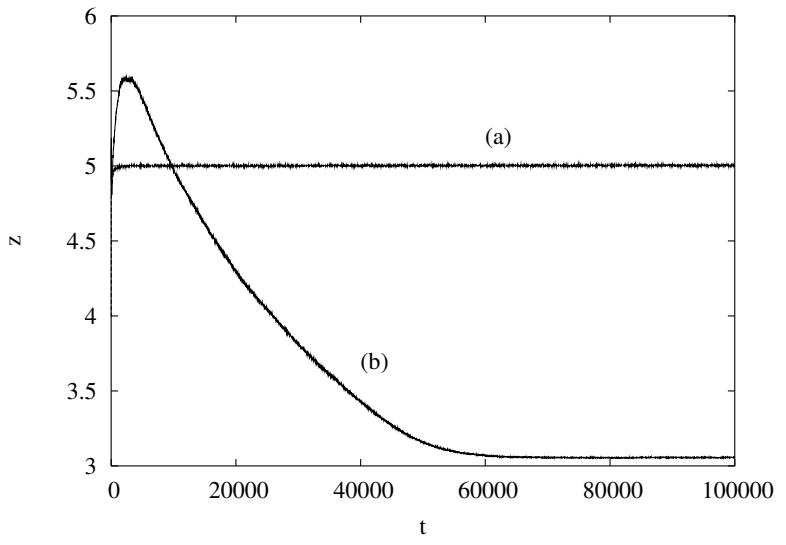

FIG. 2: Time dependence of the global fitness $z$ for simulations with the initial state such that (a) $0<p_{i}<1$ and (b) $0<p_{i}<0.1$. Simulations were done for $m=0.3$ and $g=4$ and results are averages of 100 independent runs.

apply to the university $(r \simeq 1)$. Consequently, in this phase the average fitness remains very close to the value $z=s m+f(1-m)=10 m$ (i.e., a value when everyone is applying), as verified by numerical calculations (upper inset of Fig. (1).

When the give-up payoff $g$ is sufficiently large or the capacity of the university $m$ is sufficiently small, struggling for admission to the university is no longer the best strategy. Indeed, we observe that in such a case the model evolves differently. Now, only a part of the population aims at the university education and has $l_{i}$ and $p_{i}$ close to unity. In addition to that there is a part of the population that receives smaller but more secure give-up payoff $g$. To receive this payoff an individual should not apply to the university and that is why in this part of the population $l_{i}$ and $p_{i}$ are quite close to zero (let us notice that according to Eq. (1) the smaller $l_{i}$ and $p_{i}$ are, the smaller probability of applying to the university is). In this stratified phase the ratio of applicants $r$ is definitely smaller than unity and the fitness $z$ is larger than in the homogeneous phase (insets of Fig. 1).

As we already mentioned, the larger the fitness of an individual the larger its survival and reproduction chances. One can thus expect that a system will evolve toward states of maximal fitness. Such an expectation originates partially from our experience with thermodynamical systems where dynamics typically drives a system toward a state that minimizes its free energy. But numerical simulations show that evolutionary systems like our model do not meet these expectations. In Fig. 2 we show the time dependence of the fitness $z$ calculated for $g=4, m=0.3$ and $N=1000$. For such values of $g$ and $m$, when the system starts with $l_{i}$ and $p_{i}$ uniformly drawn from the interval $(0,1)$ it evolves toward the stratified phase (see Fig. (1). We checked that there is a lot of other initial conditions (i.e., ways to draw initial values of $l_{i}$ and $p_{i}$ ) that 

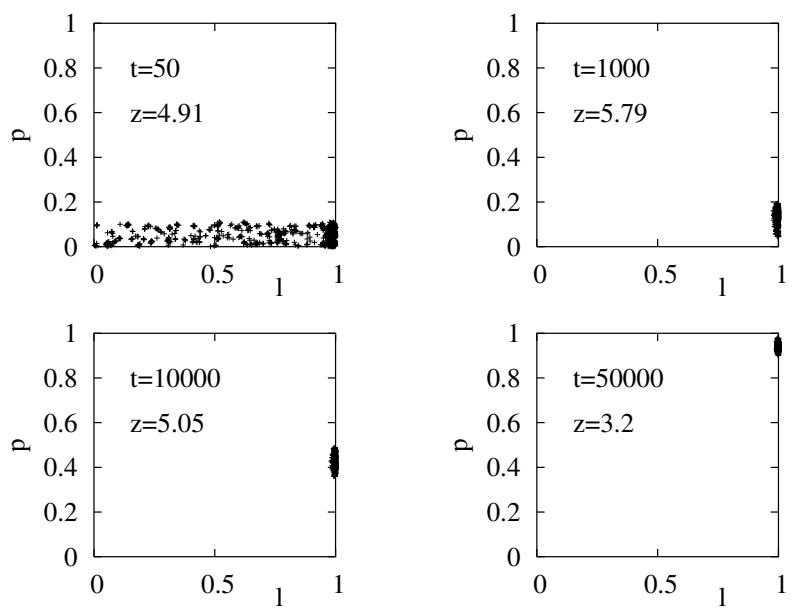

FIG. 3: Snapshots of distributions of $l_{i}$ and $p_{i}$ for simulations with the initial state such that $0<p_{i}<0.1$. Simulations were done for $m$ and $g$ as in Fig. 2 For $t=1000$ the system is very close to the state with maximal fitness $z=5.8$

lead to the same phase. However, some initial conditions yields a different behaviour. In particular, when $p_{i}$ are initially drawn from a smaller interval namely, $(0,0.1)$, the system evolves toward the homogeneous phase with all $l_{i}$ and $p_{i}$ close to unity (Fig. 2] Fig. 3). Qualitative understanding of such a behaviour is as follows: When individuals have small values of $p_{i}$, the number of individuals that apply to the university is also small, and in particular smaller than the capacity of the university $M$. As a result, nobody gets the failure payoff $f$ and the population consists only of individuals with payoffs $s$ and $g$. Since $s>g$ individuals who apply to the university gain some evolutional advantage. Of course, under such conditions individuals with larger $p_{i}$ and $l_{i}$ are preferred since for them probability to apply to the university is larger, and individuals with small $p_{i}$ and $l_{i}$ are depleted. During such an evolution the fraction of people applying to the university $r$ increases (that leads to the increase of the average fitness $z$ (Fig. [2) ) and at a certain moment the population reaches the point where $r=m$. At this point the population has the largest possible fitness $z_{M}=s m+g(1-m)$ that in our case $s=10, g=4$ and $m=0.3$ yields $z_{M}=5.8$ [ $]$ ]. However, the evolution does not stop here but drives the system towards the homogeneous phase with much smaller fitness $z$ (Fig. 2). Let us also notice that even in the stratified phase the fitness $z(\sim 5.0)$ is lower (although not that much) than the maximal value $z_{M}$.

An important characteristic that can be extracted from our model is an average number of applicants $r$ divided by the capacity of the university $m$. This is actually the number of applicants per seat and it is an obvious measure of competition for the admission. In Fig. 4 we present numerical calculation of this quantity.

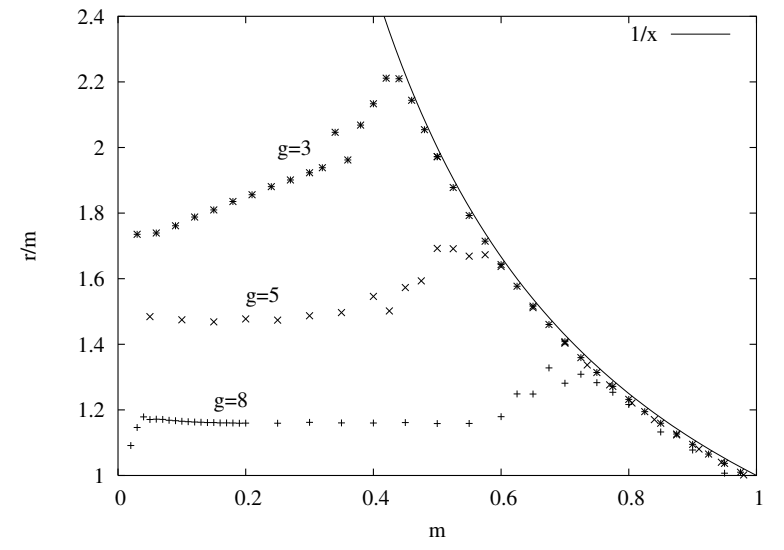

FIG. 4: The number of applicants per seat as a function of the capacity of the university $m$ calculated for several values of $g$.

First, one can see that in the homogeneous phase $r / m$ is well approximated with the function $1 / m$, which is an straightforward consequence of the fact that in this phase probability of applying to the university is very large and hence $r$ is very close to unity. The behaviour dramatically changes in the stratified phase where the number of applicants per seat $r / m$ decreases when the capacity $m$ decreases. Apparently, a decreased capacity makes an admission less likely and that discourages potential applicants. Moreover, one can see that a maximum of $r / m$ that corresponds to the most fierce competition among candidates occurs most likely at the transition between stratified and homogeneous phases.

Although the number of applicants per seat decreases, for $g=3$ and 5 it is still definitely larger than unity. As a result the university has a plenty of candidates to chose from, and the level of university students $\bar{l}$ is very close to unity. An indication of a possibly different behaviour is seen in Fig. 4 for $g=8$ and very small $m$ (where a sharp drop of $r / m$ is seen). Indeed, in this case, the average level $\bar{l}$ is definitely smaller than unity (inset of Fig. [5). To explain such a behaviour we looked in more details at individuals accepted to the university. It turns out that for small $m$ a non negligible fraction of successful applicants has quite a low level $l_{i}$ (Fig. 15). Let us recall that for such values of $g$ and $m$ the system is in the stratified phase. Typically, even in the stratified phase successful applicants belong only to that part of the population where $l_{i}$ and $p_{i}$ are very close to unity. However, Fig. 5 shows that for $g$ close to $s$ and small $m$ the other part of the population (i.e., that with $l_{i}$ and $p_{i}$ close to zero) gets access to the university as well. Although it reduces the level of applicants $\bar{l}$, in a wider social context it might be a desirable feature that candidates from other (less educated) group can enter such an élite university.

Finally, let us notice that certain features of our model bear some similarity to minority games [7]. Indeed, the 


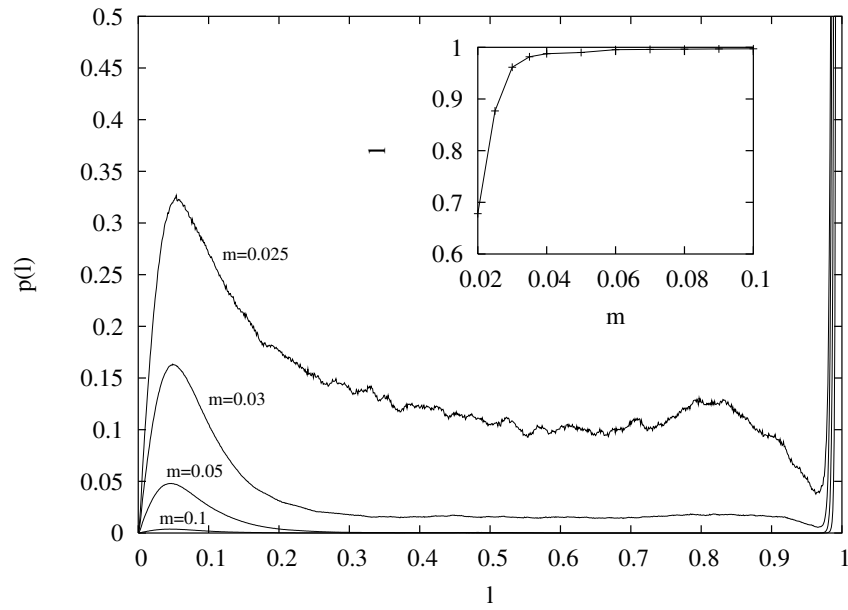

FIG. 5: Probability distributions for accepted individuals to have the level $l$ calculated for $g=8$ and several values of $m$. Inset shows the average level $\bar{l}$ as a function of $m$ for $g=8$

payoff of an individual in our model depends on the behaviour of other individuals. Moreover, individuals have a one-step memory and remember the average level $\bar{l}$ of successful applicants from the previous enrollment. Although our model seems to be more complicated, we hope that it might be interesting to examine further similarities with minority games since the later ones were already subject of intensive research and are much better understood. For example on can study a version of our model where individuals have a longer memory and see how performance of an individual depends on its memory. Another possible generalization might be to examine the case of two universities (with e.g., different success payoffs $s$ ) and/or the role of spatial effects. Such effects are known to play an important role in the evolutionary versions of prisoner's dilemma model [8].

Nevertheless, the behaviour of our model seems to be to some extent generic. Indeed, simulating the model with probability of applying to the university being independent of $l_{i}$ and given as $h\left(l_{i}, p_{i}\right)=p_{i}$ we also observed e.g., the existence of stratified and homogeneous phases. However, such a choice of $h\left(l_{i}, p_{i}\right)$ we consider as less realistic. Moreover, these phases appear also for all $p_{i}$ equal to unity (and kept constant). However, some other effects reported in this paper do not appear in this case.

In summary we introduced an evolutionary, multiagent model of an enrollment process, where a population of individuals of different levels of abilities $l_{i}$ and confidence $p_{i}$ have to decide whether to apply or not to the university. Depending on their decisions (and decisions of all other individuals) they receive certain payoffs. Such a payoff determines fitness of a given individual and that in turn determines its chance of survival and reproductivity. Numerical simulations show that depending on parameters (payoff values or capacity of the university) the system might be in either a homogeneous phase, with all individuals having $l_{i}$ and $p_{i}$ close to unity (i.e., taking almost maximal values ) or in the stratified phase where a part of the population has much lower values of $l_{i}$ and $p_{i}$. Although dynamics of the model favors individuals with a large fitness under certain conditions the model is driven toward states where the global fitness of all individuals is quite low. Even more, on the way to such states it passes through states of large fitness, that unfortunately cannot trap it. This result is actually quite worrying and perhaps not unrealistic. It shows that a society might be driven toward an undesirable state even though individuals are making no explicit efforts to enter such a state. Moreover, our simulations show that decreasing the capacity of the university the number of candidates per seat increases but only in the homogeneous phase. When this capacity is too low the model enters a stratified phase and the number of candidates per seat decreases (apparently put off by a large risk of failure). In the extreme case of a very small capacity, competition drops even further and as a result applicants of a quite poor abilities (from a different social group) can succeed.

Acknowledgements: The research grant 1 P03B 01427 from KBN is gratefully acknowledged. Numerical calculations were partially performed on ' Open Mosix Cluster' built and administrated by dr. L. Dȩbski.

[1] E. Sklar, M. Davies, and M. S. T. Co, in Proceedings of $A A M A S-2004$, N. Jennings, C. Sierra, L. Sonenberg, and M. Tambe (eds.) p. 998. R. Ganeshan, W. L. Johnson, and E. Shaw, Pedagogical Agents on the Web, in Proceedings of the Third International Conference on Autonomous Agents, p.283-290 (1999). J. E. Greer and G. I. M. eds, Student Models: The Key to Individualized Educational Systems (Springer Verlag, New York 1994).

[2] R. Axelrod and W. D. Hamilton, Science 211, 1390 (1981). K. Sigmund, Games of Life, (Oxford Univ. Press, 1993).

[3] R.Axelrod, The evolution of cooperation, (Basic Books, New York 1984).

[4] Parameters $l_{i}$ and $p_{i}$ of an offspring are given as $l_{i}=l_{i}^{p}+\omega$, $p_{i}=p_{i}^{p}+\omega$, where $\omega$ is a randomly chosen number such that $-\delta / 2<\omega<\delta / 2$ and $l_{i}^{p}$ and $p_{i}^{p}$ are parent parameters.

[5] A Java applet that simulates our model and illustrates its qualitative behaviour is available at: http://spin.amu.edu.pl/ lipowski/student.html

[6] Since the point where $r=m$ for different runs is reached at slightly different times, after averaging we obtain in Fig. 2] a peak of $z$ at a slightly smaller value than 5.8.

[7] D. Challet, M. Marsili, and R. Zecchina, Phys. Rev. Lett. 84, 1824 (2000). M. Marsili, R. Mulet, F. Ricci-Tersenghi, and R. Zecchina, Phys. Rev. Lett. 87, 208710 (2001). M. Sysi-Aho, A. Chakraborti, and K. Kaski, Phys. Rev. E 69, 036125 (2004).

[8] M.Nowak and R. M. May, Science 359, 826 (1992). 